\title{
TIME-DELAY ESTIMATION IN A SPATIALLY STRUCTURED MODEL USING DECOUPLED ESTIMATORS FOR TEMPORAL AND SPATIAL PARAMETERS
}

\author{
Felix Antreich*, Josef A. Nossek ${ }^{\star}$, Gonzalo Seco ${ }^{\dagger}$, and A. Lee Swindlehurst ${ }^{\ddagger}$ \\ German Aerospace Center (DLR), Inst. for Communications and Navigation, 82234 Wessling, Germany, phone: +49815328 2119 * \\ Inst. for Circuit Theory \& Signal Processing, Munich University of Technology (TUM), Germany ${ }^{\star}$ \\ Dept. of Telecommunications \& Systems Engineering, Universitat Autnoma de Barcelona (UAB), Spain ${ }^{\dagger}$ \\ Dept. of Electrical Engineering \& Computer Science, The University of California, Irvine CA, U.S.A. ${ }^{\ddagger}$ \\ felix.antreich@dlr.de*, nossek@nws.ei.tum.de*, gonzalo.seco@uab.es ${ }^{\dagger}$, swindle@uci.edu $^{\ddagger}$
}

\begin{abstract}
This paper deals with the joint estimation of time-delay and spatial (direction-of-arrival, DOA) parameters of several replicas of a known signal in an unknown spatially correlated field. Unstructured and structured models have been proposed in the literature. The former suffers from a severe performance degradation in some scenarios, whereas the latter involves huge complexity. It is shown how the extended invariance principle (EXIP) can be applied to obtain estimates with the quality of those of the structured model, but with the complexity of the unstructured one. We present a method to improve the quality of the time-delay estimates obtained with an unstructured spatial model when an estimate of the DOAs is available. Exemplarily, simulation results for time-delay estimation for GPS (Global Positioning System) are included and confirm that our proposal approaches the Cramer-Rao lower bound (CRLB) of the structured model even when suboptimal DOA estimates obtained by ESPRIT are introduced.
\end{abstract}

\section{INTRODUCTION}

Channel estimation is important in many applications as MIMO channel characterization, radar, synchronization, and Global Navigation Satellite Systems (GNSS) like GPS (Global Positioning System). A simple unstructured data model has been used for such problems in order to have low complexity [1] and a solution for an unknown spatial field was given in [2]. On the other hand the structured data model provides better results $[3,4]$ but has a high complexity and only seems to be easily manageable in the single path case [5]. The latter work already shows how the extended invariance principle (EXIP) can be applied to refine estimates for an unstructured model to achieve the performance available using a structured model.

This work extends the approach given in [5] to a multipath case. Applying EXIP, we derive an estimator that only depends on the directions-of-arrival (DOAs) of the signals. We attain a closed form solution that allows to correct the delay estimates of the unstructured model once DOA estimates are available. In this work we will apply Unitary ESPRIT [6] for the DOA estimation. This two-step approach offers large flexibility since it is shown that an improvement of the delay estimates can be achieved even when non-maximum likelihood (ML) estimates are introduced. This enables to use various rotational invariance techniques to obtain DOA estimates and still approach the Cramer-Rao lower bound (CRLB) of the time-delay for the structured model. An important contribution of this work with respect to previous work [7] is the comparison between the accuracies of the corrected time-delay estimates after the DOA estimates obtained with Unitary ESPRIT and on the other hand the ML EXIP DOA estimates were introduced. Further, the results of the DOA estimation with Unitary ESPRIT and ML EXIP are presented and discussed.

In this work we apply this two-step approach for timedelay estimation of the line-of-sight signal (LOSS) for GPS. Here, precise time-delay estimation is needed for synchronization and thus accurate positioning, even under presence of severe multipath and interference.

\section{DATA MODEL}

We assume that $L$ narrowband planar wavefronts, $1 \leq \ell \leq L$ are impinging on an array of $M$ isotropic sensor elements. The noise-plus-interference corrupted baseband signal at the antenna output $\mathbf{y}(t) \in \mathbb{C}^{M \times 1}$ can be modelled as a superposition of $L$ wavefronts and additional temporally white Gaussian noise $\mathbf{n}(t) \in \mathbb{C}^{M \times 1}$, with zero-mean and unknown spatial covariance matrix $\mathbf{Q} \in \mathbb{C}^{M \times M}$.

\subsection{Structured Model}

For the structured data model we define the parameter vector $\boldsymbol{\theta}=\left[\operatorname{Re}\{\boldsymbol{\gamma}\}^{\mathrm{T}}, \operatorname{Im}\{\boldsymbol{\gamma}\}^{\mathrm{T}}, \boldsymbol{\phi}^{\mathrm{T}}, \boldsymbol{\tau}^{\mathrm{T}}\right]^{\mathrm{T}}$ on the domain $D_{\boldsymbol{\theta}}$ with the vector of complex amplitudes $\gamma=\left[\gamma_{1}, \ldots, \gamma_{\ell}, \ldots, \gamma_{L}\right]^{\mathrm{T}}$, the vector of azimuth angles $\phi=\left[\phi_{1}, \ldots, \phi_{\ell}, \ldots, \phi_{L}\right]^{\mathrm{T}}$, and 
the vector of time-delays $\tau=\left[\tau_{1}, \ldots, \tau_{\ell}, \ldots, \tau_{L}\right]^{\mathrm{T}}$. Thus we can write

$$
\mathbf{y}(t)=\sum_{\ell=1}^{L} \mathbf{s}_{\ell}(t)+\mathbf{n}(t),
$$

where $\mathbf{s}_{\ell}(t)$ is given by

$$
\mathbf{s}_{\ell}(t)=\mathbf{a}_{\ell}\left(\phi_{\ell}\right) \gamma_{\ell} c\left(t-\tau_{\ell}\right) .
$$

Here, $\mathbf{a}_{\ell}\left(\phi_{\ell}\right)$ denotes the steering vector of an antenna array and $c\left(t-\tau_{\ell}\right)$ denotes the pseudo-random-noise (PN) sequence with delay $\tau_{\ell}$.

\subsection{Unstructured Model}

For the simpler unstructured model we define the parameter vector $\boldsymbol{\xi}=\left[\operatorname{Re}\{\operatorname{vec}\{\mathbf{H}\}\}^{\mathrm{T}}, \operatorname{Im}\{\operatorname{vec}\{\mathbf{H}\}\}^{\mathrm{T}}, \boldsymbol{\tau}^{\mathrm{T}}\right]^{\mathrm{T}}$ on the domain $D_{\xi}$ with the matrix of spatial signatures $\mathbf{H}=$ $\left[\mathbf{h}_{1} \cdots \mathbf{h}_{\ell} \cdots \mathbf{h}_{L}\right] \in \mathbb{C}^{M \times L}$. Here, $\operatorname{vec}\{\cdot\}$ denotes the vec operator, which vectorizes a matrix by stacking its columns. Thus we can alternatively write

$$
\mathbf{s}_{\ell}(t)=\mathbf{h}_{\ell} c\left(t-\tau_{\ell}\right) .
$$

\section{MAXIMUM LIKELIHOOD (ML) ESTIMATION}

The spatial observations are collected at $N$ time instances, as $\mathbf{y}[n]=\mathbf{y}\left(n \cdot T_{s}\right)$ with $n=1,2, \ldots, N$. The channel parameters are assumed constant during the observation interval. Collecting the samples of the observation interval leads to

$$
\begin{aligned}
\mathbf{Y} & =[\mathbf{y}[1], \mathbf{y}[2], \ldots, \mathbf{y}[N]] \in \mathbb{C}^{M \times N} \\
\mathbf{N} & =[\mathbf{n}[1], \mathbf{n}[2], \ldots, \mathbf{n}[N]] \in \mathbb{C}^{M \times N} \\
\mathbf{S}(\boldsymbol{\xi}) & =[\mathbf{s}[1], \mathbf{s}[2], \ldots, \mathbf{s}[N]] \in \mathbb{C}^{M \times N}
\end{aligned}
$$

Thus, the unstructured signal model can be written in matrix notation

$$
\mathbf{Y}=\mathbf{S}(\boldsymbol{\xi})+\mathbf{N}=\sum_{\ell=1}^{L} \mathbf{S}_{\ell}\left(\boldsymbol{\xi}_{\ell}\right)+\mathbf{N}=\mathbf{H} \mathbf{C}+\mathbf{N}
$$

Here, $\boldsymbol{\xi}_{\ell}=\left[\operatorname{Re}\left\{\mathbf{h}_{\ell}\right\}^{\mathrm{T}}, \operatorname{Im}\left\{\mathbf{h}_{\ell}\right\}^{\mathrm{T}}, \tau_{\ell}\right]^{\mathrm{T}}$ contains the parameters of one wave, and $\mathbf{C}=\left[\mathbf{c}\left(\tau_{1}\right) \cdots \mathbf{c}\left(\tau_{\ell}\right) \cdots \mathbf{c}\left(\tau_{L}\right)\right]^{\mathrm{T}} \in$ $\mathbb{R}^{L \times N}$ contains the sampled PN sequence for each impinging wavefront $\mathbf{c}\left(\tau_{\ell}\right)$. The negative log-likelihood function for the unstructured data model, neglecting additive constants is [5]

$$
\begin{aligned}
\Lambda(\boldsymbol{\xi}, \mathbf{Q}) & =N \cdot \log (\operatorname{det}(\mathbf{Q})) \\
& +\operatorname{tr}\left(\mathbf{Q}^{-1}(\mathbf{Y}-\mathbf{S}(\boldsymbol{\xi}))(\mathbf{Y}-\mathbf{S}(\boldsymbol{\xi}))^{\mathrm{H}}\right),
\end{aligned}
$$

where $(\cdot)^{\mathrm{H}}$ denotes complex conjugate transposition, $\operatorname{det}(\cdot)$ the determinant operation, and $\operatorname{tr}(\cdot)$ the trace operator. The ML estimate for $\mathbf{Q}$ is given by

$$
\hat{\mathbf{Q}}=\frac{1}{N}(\mathbf{Y}-\mathbf{S}(\boldsymbol{\xi}))(\mathbf{Y}-\mathbf{S}(\boldsymbol{\xi}))^{\mathrm{H}} .
$$

When (9) is substituted into (8), the concentrated criterion is obtained:

$$
\begin{aligned}
\Lambda(\boldsymbol{\xi}) & =N \cdot \log \left(\operatorname{det}\left(\frac{1}{N}(\mathbf{Y}-\mathbf{S}(\boldsymbol{\xi}))(\mathbf{Y}-\mathbf{S}(\boldsymbol{\xi}))^{\mathrm{H}}\right)\right) \\
& +M \cdot N .
\end{aligned}
$$

\section{SPACE-ALTERNATING GENERALIZED EXPECTATION MAXIMIZATION (SAGE) ALGORITHM}

In order to obtain the ML estimates $\hat{\xi}$ for the unstructured model we apply an iterative method, the SAGE algorithm [4]. We assume that $L$ is given. The expectation step (E-Step) can be expressed as

$$
\begin{aligned}
\hat{\mathbf{X}}_{\ell} & =\mathbf{Y}-\sum_{\substack{\ell^{\prime}=1 \\
\ell^{\prime} \neq \ell}}^{L} \mathbf{S}_{\ell^{\prime}}\left(\hat{\boldsymbol{\xi}}_{\ell^{\prime}}\right), \\
\hat{\mathbf{Q}} & =\frac{1}{N}(\mathbf{Y}-\mathbf{S}(\hat{\boldsymbol{\xi}}))(\mathbf{Y}-\mathbf{S}(\hat{\boldsymbol{\xi}}))^{\mathrm{H}},
\end{aligned}
$$

and for the maximization step (M-Step) we get

$$
\begin{aligned}
& \hat{\tau}_{\ell}=\arg \max _{\tau_{\ell}}\left\{\left\|\hat{\mathbf{Q}}^{-\frac{1}{2}} \hat{\mathbf{X}}_{\ell} \mathbf{c}^{*}\left(\tau_{\ell}\right)\right\|_{2}^{2}\right\}, \\
& \hat{\mathbf{h}}_{\ell}=\frac{\hat{\mathbf{X}}_{\ell} \mathbf{c}^{*}\left(\hat{\tau}_{\ell}\right)}{N} .
\end{aligned}
$$

The parameters of each wavefront are estimated sequentially. The E-step and the M-step are performed iteratively for each wavefront until the algorithm converges. Instead of solving a $L$-dimensional non-linear optimization problem only 1 dimensional optimization procedures need to be solved. Initialization of the SAGE algorithm is carried out by successive interference cancellation starting with $\hat{\xi}=[0, \ldots, 0]^{\mathrm{T}}$ as described in [3]. The initial estimate for $\mathbf{Q}$ is

$$
\hat{\mathbf{Q}}=\frac{1}{N} \mathbf{Y} \mathbf{Y}^{\mathrm{H}}
$$

\section{EXTENDED INVARIANCE PRINCIPLE (EXIP)}

As a second step we invoke the EXIP $[8,5]$ in order to refine the ML estimates, $\hat{\xi}$, come close to the performance using an ML estimate for the structured model, $\hat{\boldsymbol{\theta}}$, for which we would need to solve a $2 \cdot L$-dimensional non-linear problem, if solved directly. We breifly recall the EXIP [5].

Assuming that there exists a function $f$ which is one to one, satisfying

$$
\boldsymbol{\xi}=f(\boldsymbol{\theta}) \in D_{\boldsymbol{\xi}} \quad, \forall_{\boldsymbol{\theta}} \in D_{\boldsymbol{\theta}}
$$

and

$$
\lim _{N \rightarrow \infty} \hat{\boldsymbol{\xi}}=\lim _{N \rightarrow \infty} f(\hat{\boldsymbol{\theta}}) .
$$


Then

$$
\hat{\hat{\boldsymbol{\theta}}}=\arg \min _{\boldsymbol{\theta}}[\hat{\boldsymbol{\xi}}-f(\boldsymbol{\theta})]^{\mathrm{T}} \mathbf{W}[\hat{\boldsymbol{\xi}}-f(\boldsymbol{\theta})],
$$

is asymptotically (for large $N$ ) equivalent to the structured ML estimate $\hat{\boldsymbol{\theta}}$, where

$$
\mathbf{W}=\left.\mathrm{E}\left\{\frac{\partial \Lambda(\boldsymbol{\xi}, \mathbf{Q})}{\partial \boldsymbol{\xi} \partial \boldsymbol{\xi}^{\mathrm{T}}}\right\}\right|_{\substack{\boldsymbol{\xi}=\hat{\boldsymbol{\xi}} \\ \mathbf{Q}=\hat{\mathbf{Q}}}},
$$

and $\mathrm{E}\{\cdot\}$ denotes expectation.

For the problem at hand we can write

$$
[\hat{\boldsymbol{\xi}}-f(\boldsymbol{\theta})]=\left[\left[\begin{array}{c}
\operatorname{Re}\{\operatorname{vec}\{\hat{\mathbf{H}}\}\} \\
\operatorname{Im}\{\operatorname{vec}\{\hat{\mathbf{H}}\}\} \\
\hat{\boldsymbol{\tau}}
\end{array}\right]-\left[\boldsymbol{\Psi}(\boldsymbol{\phi})\left[\begin{array}{c}
\operatorname{Re}\{\boldsymbol{\gamma}\} \\
\operatorname{Im}\{\boldsymbol{\gamma}\} \\
\boldsymbol{\tau}
\end{array}\right]\right],\right.
$$

where

$$
\boldsymbol{\Psi}(\boldsymbol{\phi})=\left[\begin{array}{cc}
\operatorname{Re}\left\{\mathbf{I}_{L} \square \mathbf{A}\right\} & -\operatorname{Im}\left\{\mathbf{I}_{L} \square \mathbf{A}\right\} \\
\operatorname{Im}\left\{\mathbf{I}_{L} \square \mathbf{A}\right\} & \operatorname{Re}\left\{\mathbf{I}_{L} \square \mathbf{A}\right\}
\end{array}\right]
$$

and, $\square$ denotes the Khatri-Rao product and $\mathbf{A}=$ $\left[\mathbf{a}_{1}\left(\phi_{1}\right) \cdots \mathbf{a}_{\ell}\left(\phi_{\ell}\right) \cdots \mathbf{a}_{L}\left(\phi_{L}\right)\right] \in \mathbb{C}^{M \times L}$. The weighting matrix $\mathbf{W}$ can be expressed as

$$
\mathbf{W}=\left[\begin{array}{ccc}
\operatorname{Re}\left\{\mathbf{W}_{1}\right\} & -\operatorname{Im}\left\{\mathbf{W}_{1}\right\} & \operatorname{Re}\left\{\mathbf{W}_{3}\right\} \\
\operatorname{Im}\left\{\mathbf{W}_{1}\right\} & \operatorname{Re}\left\{\mathbf{W}_{1}\right\} & \operatorname{Im}\left\{\mathbf{W}_{3}\right\} \\
\operatorname{Re}\left\{\mathbf{W}_{3}\right\}^{\mathrm{T}} & \operatorname{Im}\left\{\mathbf{W}_{3}\right\}^{\mathrm{T}} & \operatorname{Re}\left\{\mathbf{W}_{2}\right\}
\end{array}\right],
$$

with

$$
\begin{aligned}
\mathbf{W}_{1}= & 2 \cdot\left(\mathbf{C ~}^{\mathrm{H}}\right) \otimes \hat{\mathbf{Q}}^{-1}, \\
\mathbf{W}_{2}= & 2 \cdot\left(\mathbf{\Upsilon}^{\mathrm{H}} \mathbf{\Upsilon}\right) \odot\left(\hat{\mathbf{H}}^{\mathrm{H}} \hat{\mathbf{Q}}^{-1} \hat{\mathbf{H}}\right), \\
\mathbf{W}_{3}= & {\left[\begin{array}{c}
2 \cdot\left(\hat{\mathbf{Q}}^{-1} \hat{\mathbf{H}}\right) \operatorname{diag}\left\{\left(\mathbf{c}^{\mathrm{H}}\left(\hat{\tau}_{1}\right) \mathbf{\Upsilon}\right\}\right. \\
\vdots \\
2 \cdot\left(\hat{\mathbf{Q}}^{-1} \hat{\mathbf{H}}\right) \operatorname{diag}\left\{\left(\mathbf{c}^{\mathrm{H}}\left(\hat{\tau}_{\ell}\right) \mathbf{\Upsilon}\right\}\right. \\
\vdots \\
2 \cdot\left(\hat{\mathbf{Q}}^{-1} \hat{\mathbf{H}}\right) \operatorname{diag}\left\{\left(\mathbf{c}^{\mathrm{H}}\left(\hat{\tau}_{L}\right) \mathbf{\Upsilon}\right\}\right.
\end{array}\right] . }
\end{aligned}
$$

Here, $\otimes$ denotes the Kronecker product, $\operatorname{diag}\{\cdot\}$ defines a diagonal matrix, and

$$
\mathbf{\Upsilon}=\left[\frac{\partial \mathbf{c}\left(\hat{\tau}_{1}\right)}{\partial \hat{\tau}_{1}} \cdots \frac{\partial \mathbf{c}\left(\hat{\tau}_{\ell}\right)}{\partial \hat{\tau}_{\ell}} \cdots \frac{\partial \mathbf{c}\left(\hat{\tau}_{L}\right)}{\partial \hat{\tau}_{L}}\right] .
$$

Thus, introducing (20) in (18) and then minimizing (18) with respect to $\tau$, for fixed $\gamma$ and $\phi$ leads to the refined time-delay estimates

$$
\begin{array}{r}
\hat{\boldsymbol{\tau}}=\hat{\boldsymbol{\tau}}+\operatorname{Re}\left\{\mathbf{W}_{2}\right\}^{-1}\left[\operatorname{Re}\left\{\mathbf{W}_{3}\right\}^{\mathrm{T}} \operatorname{Im}\left\{\mathbf{W}_{3}\right\}^{\mathrm{T}}\right] \\
\cdot\left[\left[\begin{array}{c}
\operatorname{Re}\{\operatorname{vec}\{\hat{\mathbf{H}}\}\} \\
\operatorname{Im}\{\operatorname{vec}\{\hat{\mathbf{H}}\}\}
\end{array}\right]-\boldsymbol{\Psi}(\boldsymbol{\phi})\left[\begin{array}{l}
\operatorname{Re}\{\boldsymbol{\gamma}\} \\
\operatorname{Im}\{\boldsymbol{\gamma}\}
\end{array}\right]\right]
\end{array}
$$

Note that the second term in (27) acts like a correction of the unstructured ML estimates $\hat{\tau}$.

Replacing (27) in (20) and then minimizing (18) with respect to $\gamma$ leads to

$$
\begin{aligned}
{\left[\begin{array}{c}
\operatorname{Re}\{\hat{\hat{\gamma}}\} \\
\operatorname{Im}\{\hat{\hat{\gamma}}\}
\end{array}\right] } & =\left(\boldsymbol{\Psi}^{\mathrm{T}}(\boldsymbol{\phi}) \boldsymbol{\Omega} \boldsymbol{\Psi}(\boldsymbol{\phi})\right)^{-1} \\
& \cdot \boldsymbol{\Psi}^{\mathrm{T}}(\boldsymbol{\phi}) \boldsymbol{\Omega}\left[\begin{array}{l}
\operatorname{Re}\{\operatorname{vec}\{\hat{\mathbf{H}}\}\} \\
\operatorname{Im}\{\operatorname{vec}\{\hat{\mathbf{H}}\}\}
\end{array}\right],
\end{aligned}
$$

where $\boldsymbol{\Omega}$ denotes the Schur complement of block $\operatorname{Re}\left\{\mathbf{W}_{2}\right\}$ in matrix $\mathbf{W}$, namely

$$
\begin{aligned}
\boldsymbol{\Omega} & =\left[\begin{array}{cc}
\operatorname{Re}\left\{\mathbf{W}_{1}\right\} & -\operatorname{Im}\left\{\mathbf{W}_{1}\right\} \\
\operatorname{Im}\left\{\mathbf{W}_{1}\right\} & \operatorname{Re}\left\{\mathbf{W}_{1}\right\}
\end{array}\right] \\
& \left.-\left[\begin{array}{c}
\operatorname{Re}\left\{\mathbf{W}_{3}\right\} \\
\operatorname{Im}\left\{\mathbf{W}_{3}\right\}
\end{array}\right] \operatorname{Re}\left\{\mathbf{W}_{2}\right\}^{-1}\left[\operatorname{Re}\left\{\mathbf{W}_{3}\right\}^{\mathrm{T}} \operatorname{Im}\left\{\mathbf{W}_{3}\right\}^{\mathrm{T}}\right]\right] .
\end{aligned}
$$

Finally, $\hat{\hat{\phi}}$ can be obtained by substituting $\tau$ and $\gamma$ in (18) for (27) and (??)

$$
\begin{gathered}
\hat{\hat{\boldsymbol{\phi}}}=\arg \max _{\boldsymbol{\phi}}\left\{\left[\begin{array}{c}
\operatorname{Re}\{\operatorname{vec}\{\hat{\mathbf{H}}\}\} \\
\operatorname{Im}\{\operatorname{vec}\{\hat{\mathbf{H}}\}\}
\end{array}\right]^{\mathrm{T}} \boldsymbol{\Omega} \boldsymbol{\Psi}(\boldsymbol{\phi})\right. \\
\left.\cdot\left(\boldsymbol{\Psi}^{\mathrm{T}}(\boldsymbol{\phi}) \boldsymbol{\Omega} \boldsymbol{\Psi}(\boldsymbol{\phi})\right)^{-1} \boldsymbol{\Psi}^{\mathrm{T}}(\boldsymbol{\phi}) \boldsymbol{\Omega}\left[\begin{array}{c}
\operatorname{Re}\{\operatorname{vec}\{\hat{\mathbf{H}}\}\} \\
\operatorname{Im}\{\operatorname{vec}\{\hat{\mathbf{H}}\}\}
\end{array}\right]\right\} .
\end{gathered}
$$

Therefore, (30) provides estimates of the DOAs that are asymptotically equivalent to the ML estimates of the structured model. The estimate $\hat{\hat{\phi}}$ is introduced in (28) and (27) improving the delay estimates. In general the maximization of (30) is complex unless particular array geometries are employed. Subspace fitting methods can be used, while maintaining the same asymptotic performance.

\section{DIRECTION OF ARRIVAL (DOA) ESTIMATION WITH UNITARY ESPRIT}

Instead of solving (30) we apply Unitary ESPRIT with structured least squares (SLS) with one iteration [6] and spatial smoothing. We use the pre-whitened channel estimate

$$
\hat{\mathbf{R}}=\hat{\mathbf{Q}}^{-\frac{1}{2}} \hat{\mathbf{H}}\left(\hat{\mathbf{Q}}^{-\frac{1}{2}} \hat{\mathbf{H}}\right)^{\mathrm{H}},
$$

to follow the covariance approach as given in [6].

Note that it is necessary to perform a pairing between the DOAs estimates and the columns of $\hat{\mathbf{H}}$, in order to apply (27). Only with the correct pairing between the DOA estimates and the parameters for the unstructured model estimated with the SAGE algorithm, EXIP can be applied properly, and thus an improvement of the delay estimates can be achieved. Let us assume now that $\hat{\hat{\phi}}$ are the estimates obtained by Unitary ESPRIT. For each of the $L$ ! permutations, we define the ma$\operatorname{trix} \hat{\hat{\mathbf{A}}}_{p}=\left[\hat{\hat{\mathbf{a}}}_{p, 1}\left(\hat{\hat{\phi}}_{p, 1}\right) \cdots \hat{\hat{\mathbf{a}}}_{p, \ell}\left(\hat{\hat{\phi}}_{p, \ell}\right) \cdots \hat{\hat{\mathbf{a}}}_{p, L}\left(\hat{\hat{\phi}}_{p, L}\right)\right]$ with 
$p=1, \ldots, L$ !. Then we solve the following least squares problem

$$
\min _{p=1, \ldots, L !} \sum_{\ell=1}^{L}\left\|\hat{\mathbf{Q}}^{-\frac{1}{2}}\left(\hat{\mathbf{h}}_{\ell}-\gamma_{p, \ell} \hat{\mathbf{a}}_{p, \ell}\right)\right\|_{2}^{2} .
$$

After eliminating $\gamma_{p, 1}, \ldots, \gamma_{p, L}$ with $\gamma_{p, \ell}=\frac{\hat{\hat{\mathbf{a}}}_{p, \ell}^{\mathrm{H}} \hat{\mathbf{Q}}^{-1} \hat{\mathbf{h}}_{\ell}}{\hat{\mathbf{a}}_{p, \ell}^{\mathrm{H}} \hat{\mathbf{Q}}^{-1} \hat{\mathbf{a}}_{p, \ell}}$ we can write

$$
\max _{p=1, \ldots, L !} \sum_{\ell=1}^{L} \frac{\left|\hat{\hat{\mathbf{a}}}_{p, \ell}^{\mathrm{H}} \hat{\mathbf{Q}}^{-1} \hat{\mathbf{h}}_{\ell}\right|^{2}}{\hat{\hat{\mathbf{a}}}_{p, \ell}^{\mathrm{H}} \hat{\mathbf{Q}}^{-1} \hat{\hat{\mathbf{a}}}_{p, \ell}} .
$$

The result of the maximization in (33) which is only dependent on the columns of $\hat{\hat{\mathbf{A}}}$, on the columns of $\hat{\mathbf{H}}$ and on $\hat{\mathbf{Q}}$, provides the permutation given by $\hat{\hat{\mathbf{A}}}_{p}$, which indicates the pairing of the DOA estimates obtained by Unitary ESPRIT and the columns of $\hat{\mathbf{H}}$. After applying the derived pairing, $\hat{\hat{\phi}}$ together with $\hat{\xi}$ can be introduced to (28) and (27), and thus this leads to a significant improvement of the delay estimates, as will be seen next.

\section{SIMULATION RESULTS}

We assume a centro-symmetric [6] uniform linear array (ULA) with $M=8$ isotropic sensor elements with halfwavelength spacing. The one-sided bandwidth of the signal is $B=1.023 \mathrm{MHz}$. For the PN sequence we apply Gold codes [9] as used for the GPS C/A code with code period $T=1 \mathrm{~ms}$, 1023 chips per code period each with a time duration $T_{c}=$ 977.52 ns. Signal-to-noise ratio (SNR) denotes the LOSS-tonoise ratio. The effective SNR in $\mathrm{dB}$ can be obtained by

$$
\mathrm{SNR}=\mathrm{C} / \mathrm{N}_{0}-10 \cdot \log _{10}(2 \cdot B)+10 \cdot \log _{10}\left(N_{c}\right),
$$

whereas $\mathrm{C} / \mathrm{N}_{0}$ in $\mathrm{dB}-\mathrm{Hz}$ denotes the carrier-to-noise density ratio and $N_{c} \in \mathbb{N}$ is the number of code periods within the observation period. We assume $\mathrm{C} / \mathrm{N}_{0}=40.3 \mathrm{~dB}-\mathrm{Hz}$ and $N_{c}=20$ which leads to $\mathrm{SNR}=-9.8 \mathrm{~dB}$. We analyze the behavior of our approach for a single reflective multipath as a function of its relative delay to the LOSS $(L=2)$. In the following parameters with the subscript 1 refer to the LOSS and parameters with the subscript 2 refer to the reflection. The reflected multipath and the LOSS are considered to be in-phase, which means $\arg \left(\gamma_{1}\right)=\arg \left(\gamma_{2}\right)$, and the signal-to-multipath ratio (SMR) is $5 \mathrm{~dB}$. Further, we assume a temporally white Gaussian interference with interference-to-signal ratio (ISR) of $40 \mathrm{~dB}$ which is responsible for the spatial covariance of the noise plus interference field. The interference is uncorrelated with the signals and the noise. The DOAs for the LOSS and the multipath are $\phi_{1}=-30^{\circ}$ and $\phi_{2}=62^{\circ}$, and for the interference is $\phi_{I}=10^{\circ}$. We define $\Delta \tau=\left|\tau_{1}-\tau_{2}\right| / T_{c}$.

For the spatial smoothing with $L=2$ we define the following selection matrices for two subarrays with maximum overlap [6]:

$$
\mathbf{J}_{1}=\left[\begin{array}{ll}
\mathbf{0} & \mathbf{I}_{M-1}
\end{array}\right] \in \mathbb{R}^{M \times M}, \mathbf{J}_{2}=\left[\begin{array}{ll}
\mathbf{I}_{M-1} & \mathbf{0}
\end{array}\right] \in \mathbb{R}^{M \times M} .
$$

Thus, we get the spatially smoothed covariance matrix

$$
\hat{\mathbf{R}}_{s s}=\frac{1}{2}\left(\mathbf{J}_{1} \hat{\mathbf{R}} \mathbf{J}_{1}^{\mathrm{T}}+\mathbf{J}_{2} \hat{\mathbf{R}} \mathbf{J}_{2}^{\mathrm{T}}\right),
$$

which is to be used by Unitary ESPRIT with SLS for the DOA estimation. In Fig. 1 a flow diagram of the proposed two-step approach is given.

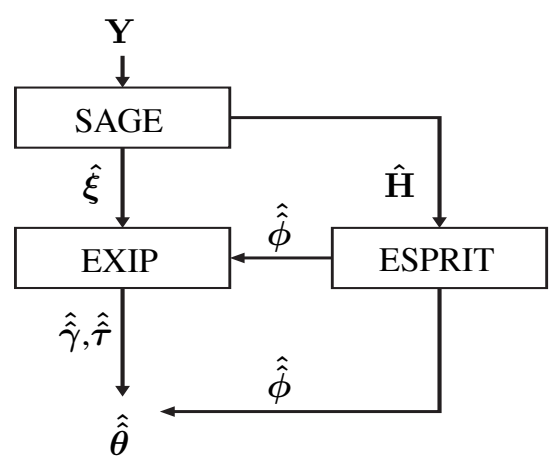

Fig. 1. Flow diagram of two-step approach

In Fig. 2 the root mean square error (RMSE) of $\hat{\tau}_{1}$ and $\hat{\hat{\tau}}_{1}$ are depicted. RMSE $\hat{\hat{\tau}}_{1}$, ESPRIT denotes the approach where the DOA estimates are obtained by Unitary ESPRIT and RMSE $\hat{\hat{\tau}}_{1}$, EXIP denotes the case where the DOA estimates are obtained by solving (30). In this work RMSE $\hat{\hat{\tau}}_{1}$, EXIP was obtained by an exhaustive, 2-dimensional search.

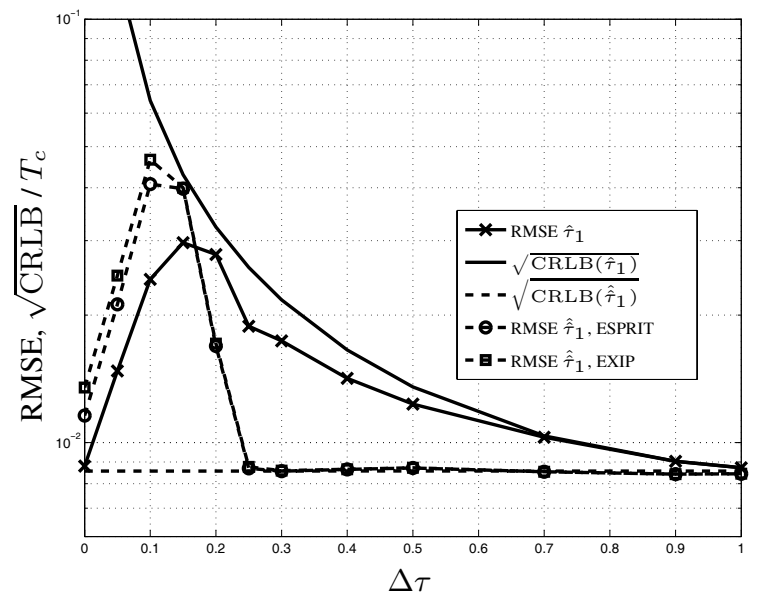

Fig. 2. RMSE of $\hat{\tau}_{1}$ and $\hat{\hat{\tau}}_{1}$ versus $\Delta \tau$

In Fig. 2 the SAGE algorithm for small $\Delta \tau$ becomes biased and the RMSE $\hat{\tau}_{1}$ is below $\sqrt{\operatorname{CRLB}\left(\hat{\tau}_{1}\right)}$, since the interference cancellation in the E-step (11) is not able to separate 
the two waves if $\Delta \tau$ is very small [4]. In these cases the estimate of one hidden data space, $\hat{\mathbf{X}}_{1}$ is an estimate for a superposition of the two wavefronts and the other, $\hat{\mathbf{X}}_{2}$ includes only noise. We used a quantization precision of 0.5 ns $\left(\approx 0.0005 \cdot T_{c}\right)$ for the time-delay estimates $\hat{\tau}$ and we set a maximum of 50 iteration cycles for the SAGE algorithm. The RMSE for the time-delay estimate $\hat{\hat{\tau}}_{1}$ shows equal behavior for the approach using Unitary ESPRIT and on the other hand for solving (30) with an exhaustive search. This indicates that the approach using Unitary ESPRIT is efficient and that the methodology to derive the correct pairing between the DOA estimates and the columns of $\hat{\mathbf{H}}$ is appropriate.

In Fig. 3 and Fig. 4 the RMSE $\hat{\hat{\phi}}_{1}$ and the RMSE $\hat{\hat{\phi}}_{2}$ for using Unitary ESPRIT or solving (30) with an exhaustive search are depicted.

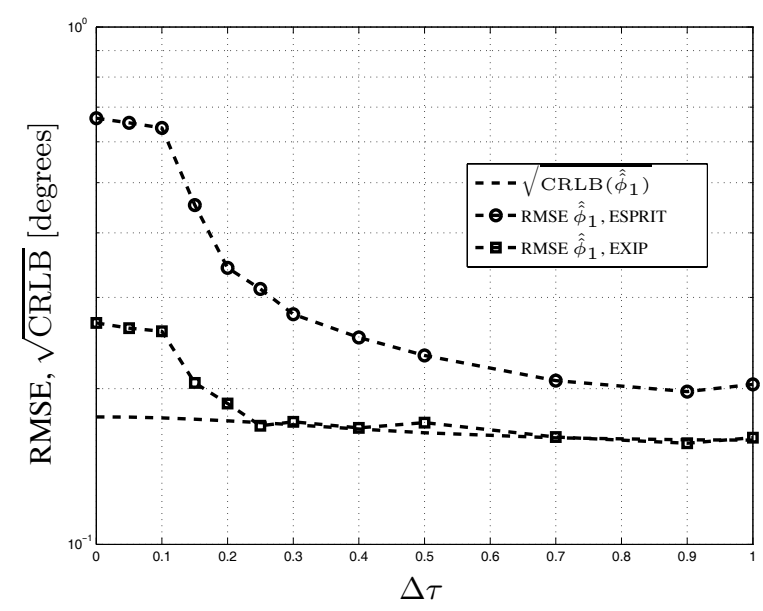

Fig. 3. RMSE of $\hat{\hat{\phi}}_{1}$ of ESPRIT and EXIP versus $\Delta \tau$

For the case where we solve (30) with an exhaustive search, the DOA of the multipath can not be resolved for $\Delta \tau<0.2$ as depicted in Fig. 4. This is consistent with the behavior of the SAGE algorithm, which is not capable of resolving the two paths for $\Delta \tau<0.2$ as described above (cf. Fig. 2). Unitary ESPRIT shows better capability to resolve the DOA of the multipath due to the use of spatial smoothing. However, this does not affect the corresponding RMSE $\hat{\hat{\tau}}$ significantly.

\section{CONCLUSIONS}

In this work we proposed a two-step approach to achieve estimates for a structured model for the multipath case in an unknown spatially colored field. We applied the EXIP to refine estimates achieved by an iterative ML estimator for an unstructured model, when an estimate of the DOAs is available. Exemplarily, simulation results for time-delay estimation in

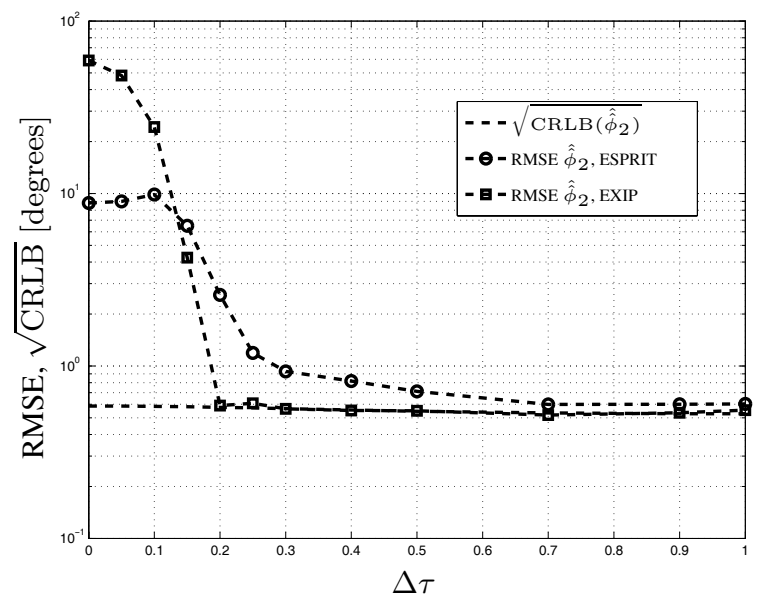

Fig. 4. RMSE of $\hat{\hat{\phi}}_{2}$ of ESPRIT and EXIP versus $\Delta \tau$

GPS are shown which confirm that the proposed two-step approach attains the CRLB of the structured model even when suboptimal DOA estimates obtained by Unitary ESPRIT are introduced.

\section{REFERENCES}

[1] A. L. Swindlehurst, "Time Delay and Spatial Signature Estimation Using Known Asynchronous Signals," IEEE Transactions on Signal Processing, vol. 46, no. 2, February 1998.

[2] G. Seco, A. L. Swindlehurst, and D. Astly, "Exploiting Antenna Arrays for Synchronization," in Signal Processing Advances in Wireless and Mobile Communications, Volume 2: Trends in Single- and Multi-User Systems, G. B. Giannakis, P. Stoica, Y. Hua, and L. Tong, Eds., pp. 403 -430. Prentice-Hall, 2000.

[3] F. Antreich, J. A. Nossek, and W. Utschick, "Maximum Likelihood Delay Estimation in a Navigation Receiver for Aeronautical Applications," Aerospace Science and Technology, doi:10.1016/j.ast.2007.06.005, 2007.

[4] B. H. Fleury, M. Tschudin, R. Heddergott, D. Dahlhaus, and K. I. Pedersen, "Channel Parameter Estimation in Mobile Radio Environments Using the SAGE Algorithm," IEEE Journal on Selected Areas in Communications, vol. 17, no. 3, March 1999.

[5] A. L. Swindlehurst and P. Stoica, "Maximum Likelihood Methods in Radar Array Signal Processing," Proceedings of the IEEE, vol. 86, no. 2, February 1998.

[6] M. Haardt, Efficient One-, Two-, and Multidimensional High-Resolution Array Signal Processing, Ph.D. thesis, Department Electrical Engineering of Munich University of Technology, 1996.

[7] F. Antreich, J. A. Nossek, G. Seco, and L. A. Swindlehurst, "The Extended Invariance Principle Applied to Joint Time-Delay, Frequency, and DOA Estimation," in Proceedings of the 33rd IEEE International Conference on Acoustics, Speech and Signal Processing ICASSP, Las Vegas, U.S.A., April 2008.

[8] P. Stoica and T. Söderström, "On Reparametrization of Loss Functions Used in Estimation and the Invariance Principle," Signal Processing, vol. 17, pp. 383-387, August 1989.

[9] B. W. Parkinson and J. J. Spilker, Global Positioning System: Theory and Applications, vol. 1, Progress in Astronautics and Aeronautics, 1996. 